\title{
Verzeichnis der lateinischen Namen der abgehandelten Gräser mit den Hinweisen auf die entsprechende Bildtafel
}

(in den Bildunterschriften der Bildtafeln weitere Hinweise)

Agropyron repens - Echte Quecke, Gemeine Quecke

Agrostis alba - Weißstraußgras

Agrostis alba v. maritima - Salzweißstraußgras

Agrostis canina - Hundsstraußgras

Agrostis gigantea - Echtes Weißstraußgras

Agrostis spica-venti - Windhalm

Agrostis stolonifera - Flechtstraußgras

Agrostis tenuis - Rotstraußgras, Sandquecke

Agrostis vulgaris - Rotstraußgras, Sandquecke

Aira flexuosa - Drahtschmiele

Aira caespitosa - Rasenschmiele

Alopecurus aequalis - Gelber Fuchsschwanz

Alopecurus agrestis - Ackerfuchsschwanz

Alopecurus fulvus - Gelber Fuchsschwanz

Alopecurus geniculatus - Geknieter Fuchsschwanz,

Knickfuchsschwanz

Alopecurus myosuroides - Ackerfuchsschwanz

Alopecurus pratensis - Wiesenfuchsschwanz

Anthoxanthum aristatum - Ackerruchgras

Anthoxanthum odoratum - Wiesenruchgras

Anthoxanthum Puelii - Ackerruchgras

Ammophila arenaria - Strandhafer

Apera spica-venti - Windhalm

Arrhenatherum elatius - Französisches Raygras, Glatthafer

Arundo phragmites - Schilfrohr

Avena elatior - Glatthafer, Französisches Raygras

Avena fatua - Flughafer, Wildhafer

Avena flavescens - Goldhafer

Avena pratensis - Trifthafer, Wiesenhafer

Avena pubescens - Flaumhafer

Avena strigosa - Sandhafer

Beckmannia eruciformis - Raupenförmige Beckmannie

Brachypodium pinnatum - Fiederzwenke

Brachypodium silvaticum - Waldzwenke
Bildtafel 1, S. 24

Bildtafel 52, S. 76

Bildtafel 52, S. 76

Bildtafel 54, S. 78

Bildtafel 52, S. 76

Bildtafel 59, S. 85

Bildtafel 52, S. 76

Bildtafel 53, S. 77

Bildtafel 53, S. 77

Bildtafel 14, S. 36

Bildtafel 3, S. 26

Bildtafel 51, S. 75

Bildtafel 61, S. 88

Bildtafel 51, S. 75

Bildtafel 50, S. 75

Bildtafel 61, S. 88 Bildtafel 49, S. 74 Bildtafel $6 / 7$, S. 29

Bildtafel $6 / 7$, S. 29

Bildtafel $6 / 7$, S. 29

Bildtafel 67, S. 93

Bildtafel 59, S. 85

Bildtafel 4, S. 27

Bildtafel 25, S. 47

Bildtafel 4, S. 27

Bildtafel 60, S. 87

Bildtafel 5, S. 28

S. 48

Bildtafel 34, S. 57

Bildtafel 60, S. 87

Bildtafel 62. S. 89

S. 10

S. 10 
Briza media - Zittergras

Bromus arvensis - Ackertrespe

Bromus erectus - Burst, Aufrechte Trespe

Bromus hordeaceus - Weiche Trespe

Bromus inermis - Grannenlose Trespe, Wehrlose Trespe

Bromus mollis - Weiche Trespe

Bromus racemosus - Traubentrespe

Bromus secalinus Roggentrespe

Bromus sterilis - Taube Trespe

Bromus tectorum - Dachtrespe

Calamagrostis canescens - Schmalblättriges Reitgras

Calamagrostis epigeios - Sandrohr

Calamagrostis lanceolata - Schmalblättriges Reitgras

Corynephorus canescens - Igelgras, Silbergras

Cynosurus cristatus - Kammgras

Dactylis glomerata - Knaulgras

Deschampsia caespitosa - Rasenschmicle

Deschampsia flexuosa - Drahtschmiele

Digitaria Ischaemum - Faden-Fingerhirse

Digitaria sanguinalis - Blutfingerhirse

Echinochloa Crus-galli - Hühnerhirse
Bildtafel 68, S. 94

Bildtafel 42, S. 66

Bildtafel 37, S. 62

Bildtafel 38, S. 63

Bildtafel 36, S. 61

Bildtafel 38, S. 63

Bildtafel 39, S. 63

Bildtafel 35 , S. 60

Bildtafel 41, S. 65

Bildtafel 40, S. 64

Bildtafel 55, S. 79

Bildtafel 56, S. 80

Bildtafel 55, S. 79

Bildtafel 10, S. 33

Bildtafel 44, S. 69

Bildtafel 2, S. 25

Bildtafel 3, S. 26

Bildtafel 14, S. 36

Bildtafel 15, S. 38

Bildtafel 16, S. 38

Bildtafel 17, S. 39

Elymus arenarius - Blauer Ifelm, Strandroggen, Strandgerste Bildtafel 66, S. 92

Eriophorum-Arten - Wollgräser

S. 99

Festuca arundinacea - Rohrschwingel

Fesluca elatior - Wiesenschwingel

Festuca ovina - Schafschwingel

Festuca ovina v. duriuscula - Schafschwingel

Festuca ovina v. capillata - Schafschwingel

Festuca pratensis - Wiesenschwingel

Festuca rubra - Rotschwingel

Festuca rubra $v$. fallax - Horstrotschwingel

Festuca rubra v. genuina - Ausläuferrotschwingel

Glyceria aquatica - Hohes Mannagras

Glyceria fluitans - Flutendes Mannagras

Glyceria maxima - Hohes Mannagras

Helictotrichon pratense - T'rifthafer, Wiesenhafer

Helictotrichon pubescens - Flaumhafer

Holcus lanatus - Wolliges Honiggras

Holcus mollis - Weiches Honiggras

Hordeum murinum - Mäusegerste

Hordeum nodosum - Roggengerste, Wiesengerste

Hordeum secalinum - Roggengerste, Wiesengerste

Koeleria-Arten - Schillergräser
Bildtafel 47 , S. 72

Bildtafel 46, S. 71

Bildtafel 11/12, S. 34

Bildtafel 11/12, S. 34

Bildtafel 11/12, S. 34

Bildtafel 46, S. 71

Bildtafel 48, S. 73

Bildtafel 48, S. 73

Bildtafel 48 , S. 73

Bildtafel 32, S. 55

Bildtafel 33, S. 56

Bildtafel 32, S. 55

S. 48

Bildtafel 34, S. 57

Bildtafel 9, S. 31

Bildtafel 8, S. 30

Bildtafel 64, S. 91

Bildtafel 65, S. 91

Bildtafel 65, S. 91 
Lolium italicum - Italienisches Raygras, Welsches Weidelgras Bildtafel 45, S. 70

Lolium multiflorum - Italienisches Raygras,

Bildtafel 45, S. 70

Welsches Weidelgras

Lolium multiflorum var. westerwoldicum - Einjähriges $\quad$ Bildtafel 45, S. 70 Weidelgras

Lolium remotum - Leinlolch

Lolium temulentum - Taumellolch

S. 131

Lolium perenne - Deutsches Weidelgras

Bildtafel 43, S. 68

Milium effusum - Waldflattergras

S. 10

Molinia coerulea - Benthalm, Pfeifengras

Bildtafel 23, S. 4.5

Nardus stricta - Bocksbart, Steifes Borstgras

Bildtafel 13, S. 35

Panicum Crus-galli - Hühnerhirse

Panicum filiforme - Faden-Fingerhirse

Panicum glaucum - Gelbe Borstenhirse

Panicum italicum - Große Kolbenhirse

Panicum miliaceum - Rispenhirse

Panicum sanguinale - Blutfingerhirse

Panicum viride - Grüne Borstenhirse

Phalaris arundinacea - Rohrglanzgras

Phalaris canariensis - Kanariengras

Phleum nodosum - Zwiebelthimothee

Phleum pratense - Timothee, Wiesenlieschgras

Phragmites communis - Schilfrohr

Poa annua - Jährige Rispe

Poa Chaixii - Bergrispe

Poa compressa - Platthalmrispe

Poa fertilis - Fruchtbare Rispe, Späte Rispe, Sumpfrispe

Bildtafel 17, S. 39

Bildtafel 15, S. 38

Bildtafel 19, S. 40

Bildtafel 20, S. 41

Bildtafel 21, S. 42

Bildtafel 16, S. 38

Bildtafel 18, S. 40

Bildtafel 57, S. 82

Bildtafel 63, S. 90

Bildtafel 58, S. 83

Bildtafel 58, S. 83

Bildtafel 25, S. 47

Bildtafel 31, S. 54

S. 48

Poa nemoralis - Hainrispe

Bildtafel 29, S. 52

Bildtafel 28, S. 51

Bildtafel 30, S. 53

Poa palustris - Fruchtbare Rispe, Späte Rispe, Sumpfrispe Bildtafel 28, S. 51

Poa pratensis - Wiesenrispe

Bildtafel 26, S. 49

Poa pratensis v. angustifolia - Schmalblättrige Wiesenrispe

Poa pratensis $v$. latifolia - Breitblättrige Wiesenrispe

Bildtafel 26, S. 49

Poa serotina - Fruchtbare Rispe, Späte Rispe, Sumpfrispe

Poa trivialis - Gemeine Rispe

Bildtafel 26, S. 49

Bildtafel 28, S. 51

Bildtafel 27, S. 50

Setaria italica - Große Kolbenhirse

Bildtafel 20, S. 41

Setaria pumila - Gelbe Borstenhirse

Setaria viridis - Grüne Borstenhirse

Sieglingia decumbens - Niederliegender Dreizahn

Bildtafel 19, S. 40

Bildtafel 18, S. 40

Sorgum sudanense - Sudangras

Bildtafel 24, S. 46

Triodia decumbens - Niederliegender Dreizahn

Bildtafel 22, S. 43

Trisetum flavescens - Goldhafer

Bildtafel 24, S. 46

Bildtafel 5, S. 28

Triticum repens - Echte Quecke, Gemeine Quecke, Quecke

Triticum repens var. aristatum

Bildtafel 1, S. 24

Bildtafel 1, S. 24

Weingaertneria canescens - Igelgras, Silbergras

Bildtafel 10, S. 33 